\title{
RESPOSTAS DE INGESTÃO E FISIOLOGICAS DE CORDEIROS ALIMENTADOS COM TORTA DE DENDÊ (ELAEIS GUINEENSIS)
}

\author{
INGESTIVE AND PHYSIOLOGICAL RESPONSES OF LAMBS FED PALM KERNEL MEAL \\ (ELAEIS GUINEENSIS)
}

\author{
Macome, F.M. ${ }^{1}$, Oliveira, R.L. ${ }^{2}$, Araujo, G.G.L. ${ }^{3}$, Barbosa, L.P. ${ }^{3}$, Carvalho, G.G.P. ${ }^{2}$, \\ Garcez Neto, A.F. ${ }^{4}$ e Silva, T.M. ${ }^{2}$
}

${ }^{1}$ Instituto de Investigação Agraria de Moçambique. Moçambique. fmmacome@yahoo.com.br ${ }^{2}$ Departamento de Produção Animal. Universidade Federal da Bahia. Brasil.

${ }^{3}$ Embrapa Semiárido. Petrolina. Brasil.

${ }^{4}$ Universidade Federal do Paraná. Palotina-PR. Brasil.

\section{PaLAVRAS ChaVE ADICIONAIS}

Alimentação. Bem-estar. Ovinos. Ócio. Ruminação.

\section{RESUMO}

Objetivou-se com este trabalho conhecer o melhor nível de inclusão da torta de dendê na dieta de cordeiros por intermédio do comportamento ingestivo, das respostas fisiológicas e bioclimatológicas. Foram utilizados 32 ovinos machos não castrados da raça Santa Inês com peso vivo inicial de $24,8 \pm 3,59 \mathrm{~kg}$, alojados em baias individuais e distribuídos em delineamento inteiramente casualizado, com quatro tratamentos: $(0 ; 6,5 ; 13,0$ e $19,5 \%$ de torta de dendê) na dieta. As dietas foram compostas de farelo de milho, farelo de soja, torta de dendê, premix mineral e feno de tifton como volumoso, sendo fornecidas duas vezes ao dia, às 9 e $16 \mathrm{~h}$, na proporção de 50:50. O período experimental foi de 80 dias, sendo 10 dias destinados á adaptação dos animais ás dietas e ao ambiente. Foi avaliado o consumo de sólidos e água e o desempenho dos animais. Os animais foram pesados no inicio e no final do experimento e foi determinado o ganho médio diário. O tempo de ingestão e a eficiência de ingestão de matéria seca seguiram comportamento quadrático, o tempo de ruminação e o consumo de matéria seca apresentaram efeito linear decrescente e o consumo de fibra em detergente neutro e eficiência de ruminação da fibra efeito linear crescente. Níveis de torta de dendê de até $19,5 \%$ na dieta não influenciam os tempos despendidos com ócio e mastigação total, freqüência respiratória, temperatura retal e temperatura superficial. Porém, a

Recibido: 4-10-10. Aceptado: 7-11-11.

\section{AdDitionAL KEYWORDS}

Feeding. Idle. Rumination. Sheep. Wellfare.

freqüência cardíaca nos dois turnos apresentou comportamento linear decrescente com adição da torta de dendê na dieta. Não houve efeito da adição da torta, peso final e ganho médio diário. A inclusão da torta de dendê em até 19,5\% afeta alguns parâmetros de comportamento ingestivo, mas não altera de forma significativa o comportamento ingestivo. Não foi verificado efeito da adição da torta de dendê na dieta no numero de períodos de ingestão, ruminação e ócio $(p>0,05)$ As variáveis ambientais durante o período experimental proporcionaram situação de desconforto térmico aos animais.

\section{SUMMARY}

The aim of this study was to know the best level of inclusion of palm kernel meal in the diet through ingestive behavior, and physiological parameters. Thirty two Santa Ines non castred male sheep, averaging $24,48 \pm 3,59 \mathrm{~kg}$ of initial body weight were studied. The animals were housed in individual pens, were distribuited in a completely randomized design with four treatments: $(0,6.5,13.0$, and $19.5 \%)$. The diets were composed of maize meal, soybean meal, palm kernel meal, mineral premix and Tifton hay as roughage, being provided twice daily, at 9:00 am and 4:00 pm at a ratio of 50:50 roughage concentrate proportion. The experimental period lasted 80 days (10days 
for diet and environmental adaptation). The intake of solids and water and performance of animals was evaluated. The animals were weighed at the beginning and end of the experiment and the average daily gain was determined. The ingestion time ( $\mathrm{min} /$ day) and efficiency of dry matter intake $(\mathrm{g} / \mathrm{h})$ showed a quadratic response; rumination time ( $\mathrm{min} /$ day) and dry matter intake (g/day) showed a negative linear effect; rumination time and consumption of dry matter showed a linear effect and the intake of neutral detergent fiber and rumination efficiency of the fiber showed an increasing linear effect. Levels of palm kernel meal up to $19.5 \%$ in the diet did not affect time spent on leisure and total chewing, respiratory frequency, rectal temperature and superficial temperature However, heart rate behavior decreased linearly with the addition of palm kernel cake in the diet There was no effect of the addition of palm kernel meal on final weight and average daily gain. The inclusion of palm kernel meal up to $19.5 \%$ affects some parameters of ingestive behavior, but does not alter significantly the ingestive behavior. Was not observed any effect of palm kernel meal addition on the number of periods of eating, ruminating or leisure $(p>0.05)$. The environmental variables during the experimental situation provided animal thermal discomfort.

\section{INTRODUÇÃO}

A torta de dendê (Elaeis guineensis), produto resultante da polpa seca do dendê após moagem e extração do seu óleo para a produção do biodiesel ou para alimentação humana (Brasil, 1998), tem sido estudada como alternativa alimentar para ruminantes.

Varios trabalhos da literatura têm relatado a importância do uso de dendê sob desempenho animal e comportamento alimentar. Segundo (Rodrigues Filho et al., 1996; Silva et al., 2008), ao estudaram o efeito do fornecimento da torta de dendê estes autores avaliaram a possibilidade de formulações de dietas com capacidade para suprir as exigências nutricionais de ruminantes.

Na tentativa de se buscar novos alimentos alternativos para nutrição animal o conhecimento da interação destes alimentos no conforto térmico e bem estar dos animais deve ser considerado.

Objetivou-se com este trabalho conhecer o melhor nível de inclusão da torta de dendê na dieta de ovinos, por intermédio da avaliação do comportamento ingestivo e das respostas fisiológicas e bioclimatológicas.

\section{MATERIALE MÉTODOS}

Este experimento foi conduzido no período de junho a setembro de 2007, no aprisco da Escola de Medicina Veterinária da UFBA, Campus de Ondina, situada em Salvador-BA. Cidade caracterizada por clima úmido, com precipitação média anual 1900 mm e umidade relativa média do ar de $81 \%$. A temperatura média é de $25,3^{\circ} \mathrm{C}$, com máxima de $28,1^{\circ} \mathrm{C}$ e mínima de $22,5^{\circ} \mathrm{C}$ (CEI, 1994). Foram utilizados 32 ovinos, machos não-castrados, da raça Santa Inês, com idade entre 4 e 6 meses e peso corporal de $24,8 \pm$ $3,59 \mathrm{~kg}$. Os animais foram vacinados, vermifugados e alojados individualmente em baias $(1,0 \times 1,0 \mathrm{~m})$ com piso suspenso de madeira ripada, bebedouros e comedouros.

O período experimental teve duração de 80 dias, incluindo 10 dias para adaptação às dietas, às instalações e ao manejo diário. Como tratamentos, foram avaliados na dieta quatro níveis de torta de dendê $(0,0 ; 6,5$ e 13,0 e $19,5 \%$ da MS). A dieta foi fornecida na forma de ração completa, duas vezes ao dia (9 e $16 \mathrm{~h})$. As sobras foram pesadas diariamente determinando o consumo, e a quantidade fornecida foi reajustada de maneira que restassem entre 10 e $20 \%$ do oferecido. Diariamente, foi fornecida água à vontade e seu consumo, medido. O concentrado foi constituído de farelo de milho, farelo de soja, premix vitamínico e mineral específico para ovinos e torta de dendê. As dietas foram formuladas segundo recomendações do NRC (1985), a relação concentrado e volumoso moído (feno de tifton 85, Cynodon spp) foi de 50:50 (tabela I).

Durante o experimento, amostras dos alimentos e sobras foram coletadas, pesadas e acondicionadas em sacos plásticos 
Tabela I. Proporção dos ingredientes (\%MS) nas dietas experimentais. (Proportion of ingredient in experimental diets).

\begin{tabular}{lcccc}
\hline & \multicolumn{5}{c}{ Torta de dendê (\%MS) } \\
& 0,0 & 6,5 & 13,0 & 19,5 \\
\hline Farelo de milho & 37,38 & 32,36 & 27,16 & 21,78 \\
Farelo de soja & 11,12 & 9,84 & 8,52 & 7,15 \\
Torta de dendê & 0,00 & 6,30 & 12,82 & 19,57 \\
Premix & 1,50 & 1,50 & 1,50 & 1,50 \\
Feno de tifton & 50,00 & 50,00 & 50,00 & 50,00 \\
\hline
\end{tabular}

${ }^{1}$ Níveis de garantia (por $\mathrm{kg}$ em elementos ativos): cálcio $120 \mathrm{~g}$; fósforo $87 \mathrm{~g}$; sódio $147 \mathrm{~g}$; enxofre 18 g; cobre $590 \mathrm{mg}$; cobalto $40 \mathrm{mg}$; cromo $20 \mathrm{mg}$; ferro $1800 \mathrm{mg}$; iodo $80 \mathrm{mg}$; manganês $1300 \mathrm{mg}$; selênio, $15 \mathrm{mg}$; zinco 3800 mg; molibdênio 300 mg; flúor máximo $870 \mathrm{mg}$; Solubilidade do fósforo $(P)$ em ácido cítrico a $2 \%$ mínimo - $95 \%$.

identificados, que foram conservados a $-10^{\circ} \mathrm{C}$. Posteriormente, estas amostras foram descongeladas, pesadas e mantidas em estufa a $55^{\circ} \mathrm{C}$, durante 72 horas. Em seguida, foram moídas em peneira de malha de $1 \mathrm{~mm}$, reservando-se $10 \%$ para posteriores análises. Os teores de MS, matéria orgânica
(MO), proteína bruta (PB), extrato etéreo (EE), cinza (CIN), nitrogênio insolúvel em detergente neutro (NIDN) e nitrogênio insolúvel em detergente ácido (NIDA) foram efetuados de acordo com os procedimentos da AOAC (1990). As análises de fibra em detergente neutro (FDN), fibra em detergente ácido (FDA), hemicelulose, celulose e lignina foram determinadas segundo metodologia de Van Soest et al. (1991) e a porcentagem de carboidratos não-fibrosos (CNF), calculado conforme equação de Sniffen et al. (1992), a fibra em detergente neutro foi corrigida para a proteina (FDNcp).

$\mathrm{CNF}(\% \mathrm{MS})=100-(\% \mathrm{CIN}+\% \mathrm{~PB}+\% \mathrm{EE}+\% \mathrm{FDNcp})$.

A composição quimica das dietas experimentais consta na tabela II. Os animais foram submetidos à avaliação visual a cada 21 dias, durante $24 \mathrm{~h}$ em intervalos de cinco minutos, para determinação do tempo despendido em ingestão, ruminação e ócio, segundo metodologia proposta por Jonhson e Combs (1991), adaptada para um período de 24 horas. As observações iniciaram-se às $6 \mathrm{~h}$ de cada dia de observação, e durante a noite foi mantida a iluminação artificial.

Tabela II. Composição química das dietas experimentais. (Chemical composition of the experimental diets).

\begin{tabular}{lcccc}
\hline & \multicolumn{3}{c}{ Torta de dendê (\%MS) } \\
& 0,0 & 6,5 & 13,0 & 19,5 \\
\hline Matéria seca (\%) & 91,34 & 91,70 & 92,12 & 92,56 \\
Matéria orgânica (\%MS) & 95,57 & 95,83 & 95,75 & 95,66 \\
Cinzas (\%MS) & 4,43 & 4,50 & 4,58 & 4,66 \\
Proteína bruta (\%MS) & 11,00 & 11,23 & 11,46 & 11,70 \\
N insolúvel em detergente ácido (\% N total) & 10,46 & 11,39 & 12,36 & 13,36 \\
N insolúvel em detergente neutro (\% N total) & 35,91 & 37,36 & 38,87 & 40,42 \\
Fibra em detergente neutro (\%MS) & 47,60 & 51,26 & 55,05 & 58,97 \\
Fibra em detergente ácido (\%MS) & 24,89 & 27,49 & 30,18 & 32,96 \\
Lignina (\%MS) & 7,52 & 8,40 & 9,30 & 10,23 \\
Celulose (\%MS) & 17,37 & 19,09 & 20,87 & 22,72 \\
Hemicelulose (\%MS) & 22,71 & 23,77 & 24,87 & 26,01 \\
Carboidratos não-fibrosos (\%MS) & 35,22 & 30,96 & 26,54 & 21,97 \\
Extrato etéreo (\%MS) & 1,72 & 2,03 & 2,34 & 2,67 \\
\hline
\end{tabular}


O comportamento ingestivo dos animais foi avaliado por observadores treinados, posicionados de forma a não incomodar os animais. As observações visuais dos animais foram realizadas três vezes ao longo do experimento, perfazendo um total de 288 observações por período.

As eficiências de ingestão (EI) e ruminação (ERU) da MS e FDN e o tempo de mastigação total (TMT min/dia) foram calculados conforme a metodologia descrita por Burger et al. (2000), por intermédio das seguintes equações:

\section{$\mathrm{EIMS}=\mathrm{CMS} / \mathrm{TI}$}

$\mathrm{EIFDN}=\mathrm{CFDN} / \mathrm{TI}$

em que:

EIMS: eficiência de ingestão de MS (g MS ingerida/ h);

CMS (g): consumo diário de matéria seca;

CFDN (g): consumo diário de FDN;

TI: tempo gasto em ingestão diariamente.

EIFDN: eficiência de ingestão de FDN (g FDN ingerida/h).

\section{ERUMS $=$ CMS/TRU}

$\mathrm{ERUFDN}=\mathrm{CFDN} / \mathrm{TRU}$

em que:

ERUMS: é eficiência de ruminação da MS (g MS ruminada/h);

TRU: tempo gasto em ruminação diariamente $(h)$; ERUFDN: eficiência de ruminação da FDN (g FDN ruminada/h);

$$
\mathrm{TMT}=\mathrm{TI}+\mathrm{TRU}
$$

em que:

TMT: é tempo de mastigação total (min/dia).

Para o monitoramento do ambiente experimental, foram instalados termohigrômetro e termômetro de globo negro para medir e registrar a temperatura ambiente (Ta), umidade relativa do ar (URA), temperatura de globo negro (TGN) e temperatura do ponto de orvalho (Tpo) e determinar o índice de temperatura do globo negro e umidade $(\mathrm{ITGU}=\mathrm{TGN}+0,36 *(\mathrm{Tpo})+41,5)$, segundo recomendações de Buffington et al. (1981). O índice de temperatura e umidade foi calculado segundo metodologia proposta por Baeta e Sousa (1997): (ITU $=\mathrm{Ta}+0,36 \mathrm{Tpo}+$ $41,2)$. O ponto de orvalho foi calculado pela fórmula $(\mathrm{TPO}=\sqrt[8]{\mathrm{UR}} / 100 *[112+(0,9 * \mathrm{~T})]+$ $(0,1 * \mathrm{~T})-112)$. As leituras das variáveis ambientais e fisiológicas foram realizadas às $9 \mathrm{e} 15 \mathrm{~h}$, duas vezes por semana, durante todo o período experimental. As respostas fisiológicas dos animais foram avaliadas por intermédio das seguintes medições: freqüência respiratória (FR), freqüência cardíaca (FC), temperatura retal (TR) e temperatura superficial (TS) obtida pelas médias de temperatura da pele do animal, aferida com termômetro infravermelho na paleta $(\mathrm{Pa})$ e no pernil $(\mathrm{Pe})$ de ambos os lados do corpo dos animais.

Os animais foram pesados no inicio de experimento apôs os dez dias de adaptação e a cada quinze dias para avaliação do desempenho, e no final do experimento também foram pesados antes do abate para determinação do peso final e foi feita avaliação do ganho de peso.

O delineamento experimental utilizado foi o inteiramente casualizado com quatro tratamentos e oito repetições. Os dados foram submetidos a análises de regressão, utilizando-se o pacote estatístico SPSS versão 13.0 (2004) e o peso inicial como cováriavel.

\section{RESULTADOSEDISCUSSÃO}

A inclusão da torta de dendê originou efeito linear decrescente para o consumo de MS, tempo de ruminação (tabela III), provavelmente a redução no tempo de ruminação pode estar associado a redução no CMS.

Segundo Van Soest (1994), o teor da fibra e a forma física da dieta são os principais fatores que afetam o tempo de ruminação e ingestão. Carvalho et al. (2004), em estudo sobre comportamento ingestivo de cabras leiteiras submetidas a dietas com farelo de 
cacau ou torta de dendê, não encontraram diferenças nos tempos despendidos em ingestão, ruminação e ócio, fato que atribuíram a semelhança no tamanho de partícula dos alimentos ao do concentrado padrão.

Nao houve efeito $(\mathrm{p}>0,05)$ da adição da torta de dendê no consumo de nutrientes digestiveis totais e para o tempo dispendido em ócio (tabela III). A adição da torta de dendê na dieta proporcionou efeito quadrático para o tempo de ingestão ( $\mathrm{min} / \mathrm{dia})$, com o valor mínimo estimado de $274 \mathrm{~min} /$ dia para um nível de 7,43\% (tabela III).

Foi verificado comportamento quadrático à medida que se adicionou torta de dendê na dieta para a eficiência de ingestão de MS (tabela III), com um valor máximo de $302,59 \mathrm{~g}$ de $\mathrm{MS} / \mathrm{h}$ para um nível de $4,01 \%$ de torta de dendê, fato que pode ser explicado pelo consumo de MS que tendeu a decrescer com adição dos níveis de torta de dendê.

A eficiência de ruminação de FDN seguiu efeito linear crescente com a adição dos níveis de torta de dendê, o que pode ser explicado em razão do aumento do consumo de FDN com a adição da torta de dendê na dieta (tabela III). Como as dietas apresentaram altos teores de FDN (tabela II), provavelmente a eficiência de ruminação foi afetada, o que refletiu na necessidade de processamento da digesta ruminal para elevar a eficiência digestiva, fato observado na eficiência de ruminação.

Não houve efeito $(p>0,05)$ da adição da torta de dendê na dieta para o tempo despendido em mastigação total, eficiência de ingestão de FDN e eficiência de ruminação de MS (tabela III).

Tabela III. Comportamento ingestivo e desempenho de cordeiros submetidos a dietas com níveis de torta de dendê. (Ingestive behaviour and performance of lambs fed diets with increasing levels of palm kernel meal).

\begin{tabular}{|c|c|c|c|c|c|c|c|}
\hline & \multicolumn{4}{|c|}{ Níveis de torta de dendê (\%MS) } & \multirow[t]{2}{*}{ CV (\%) } & \multirow[t]{2}{*}{ Equação de regressão } & \multirow[t]{2}{*}{$\mathrm{R}^{2}$} \\
\hline & 0,00 & 6,50 & 13,00 & 19,50 & & & \\
\hline CMS & 1440,6 & 1241,8 & 1411,1 & 1200,9 & 20,80 & $Y=1406,1-8,61 X$ & 0,58 \\
\hline CFDN & 564,27 & 589,41 & 622,15 & 662,51 & 21,14 & $Y=597,42+5,56 X$ & 0,50 \\
\hline CNDT & 0,868 & 0,769 & 0,854 & 0,720 & 14,61 & $Y=0,802$ & - \\
\hline $\mathrm{TI}$ & 294,31 & 278,95 & 284,17 & 324,48 & 15,92 & $Y=294,3-4,83 X+0,3254 X^{2}$ & 0,99 \\
\hline Ocio & 607,08 & 642,92 & 635,83 & 615,20 & 12,74 & $Y=625,42$ & - \\
\hline TRU & 539,38 & 518,13 & 520,00 & 500,32 & 12,05 & $Y=536,42-1,769 X$ & 0,86 \\
\hline EIMS & 305,52 & 278,18 & 302,12 & 226,36 & 28,65 & $Y=297,97+2,301 X-0,2865 X^{2}$ & 0,71 \\
\hline EIFDN & 123,71 & 120,68 & 148,92 & 121,62 & 27,15 & $Y=127,98$ & - \\
\hline TMT & 826,04 & 797,08 & 803,33 & 822,92 & 9,72 & $Y=812,34$ & - \\
\hline ERUMS & 161,79 & 145,17 & 164,87 & 145,15 & 24,23 & $Y=154,24$ & - \\
\hline ERUFDN & 65,62 & 62,67 & 79,64 & 78,28 & 26,17 & $Y=63,39+0,842 X$ & 0,67 \\
\hline PVI & 24,47 & 24,53 & 25,13 & 23,78 & 14,66 & $Y=24,48$ & \\
\hline PVF & 37,51 & 35,92 & 37,47 & 35,52 & 10,98 & $Y=36,60$ & - \\
\hline GMD & 0,177 & 0,157 & 0,176 & 0,168 & 18,93 & $Y=0,170$ & - \\
\hline
\end{tabular}

CMS: consumo de matéria seca (g/dia); CFDN: consumo de fibra em detergente neutro (g/dia); CNDT: consumo de NDT (kg/dia); TI: tempo de ingestao ( $\mathrm{min} / \mathrm{dia})$; TRU: tempo de ruminacao ( $\mathrm{min} / \mathrm{dia})$; EIMS: eficiência de ingestão de matéria seca ( $\mathrm{g} / \mathrm{h}$ ); EIFDN: eficiencia de ingestao de fibra em detergente neutro ( $\mathrm{g}$ FDN/h); TMT: tempo de mastigação total ( $\mathrm{min} / \mathrm{dia})$; ERUMS: eficiencia de ruminação de matéria seca (g MS/h); ERUFDN: eficiência de ruminação de fibra em detergente neutro (g/h); PVI: peso vivo inicial (kg); PVF: peso vivo final (kg); GMD: ganho médio diário (kg). 
MACOME, OLIVEIRA, ARAUJO, BARBOSA, CARVALHO, GARCEZNETOE SILVA

Tabela IV. Número de períodos e tempo gasto por período de ingestão, ruminação e ócio (min) de cordeiros submetidos a dietas com níveis de torta de dendê. (Number of periods and time spent per period of eating, ruminating and idle $(\mathrm{min})$ in lambs fed diets with increasing levels of palm kernel meal).

\begin{tabular}{|c|c|c|c|c|c|c|c|}
\hline & \multicolumn{4}{|c|}{ Níveis de torta de dendê (\%MS) } & \multirow[t]{2}{*}{$\mathrm{CV}(\%)$} & \multirow[t]{2}{*}{ Equação de regressão } & \multirow[t]{2}{*}{$\mathrm{R}^{2}$} \\
\hline & 0,0 & 6,5 & 13,0 & 19,5 & & & \\
\hline \multicolumn{8}{|c|}{ Numero de períodos por dia } \\
\hline Ingestão & 17,17 & 14,63 & 15,33 & 15,88 & 29,10 & $Y=15,75$ & - \\
\hline Ruminação & 25,46 & 25,29 & 26,46 & 24,60 & 22,03 & $Y=25,42$ & - \\
\hline Ócio & 36,79 & 35,04 & 36,88 & 36,38 & 17,83 & $Y=36,27$ & - \\
\hline \multicolumn{8}{|c|}{ Tempo gasto por período (min) } \\
\hline Ingestão & 17,59 & 20,69 & 19,80 & 22,82 & 33,65 & $Y=18,00+0,2278$ & 0,77 \\
\hline Ruminação & 22,16 & 21,68 & 20,65 & 21,02 & & $Y=21,36$ & - \\
\hline Ócio & 17,50 & 18,74 & 17,70 & 14,21 & 24,66 & $Y=17,49+0,3779 X-0,02799 X^{2}$ & 0,99 \\
\hline
\end{tabular}

Apesar de ter havido efeito para os tempos de ingestão e ruminação, o tempo total de mastigação (TMT, min/dia) não foi afetado pela utilização da torta de dendê na dieta dos animais.

Não foi observada diferença significativa para números de períodos de ingestão, ruminação e ócio ( $\mathrm{n}$ / dia) e tempo gasto por período de ruminação (tabela IV).

O valor médio de 15,75 ingestões por dia (tabela IV) foi superior aos valores de 14,80 13,10 e 12,00 ingestões obtidos, respectivamente, por Burger et al.(2000) em estudo com bezerros, Carvalho et al. (2004), estudando comportamento ingestivo de cabras alimentadas com farelo de cacau ou torta de dendê e Carvalho et al. (2006), em estudo com ovinos.

$\mathrm{O}$ número de períodos de ruminação observado nesta pesquisa (tabela IV), foi superior ao encontrado por Carvalho et al. (2004), visto que a distribuição da ruminação é influenciada pela alimentação, acontece logo apos a ingestão.

A adição da torta de dendê proporcionou efeito linear crescente $(p<0,05)$ para o tempo gasto por período de ingestão e comportamento quadrático para o tempo gasto no período de ócio (tabela IV), o que pode ser explicado pela composição da dieta experi- mental que continha teores elevados de FDN em função dos níveis de torta de dendê utilizados.

Segundo os resultados do ITGU e do ITU, observou-se que os animais nos dois períodos foram expostos a situação de desconforto térmico, fato que pode ser explicado pela condição de alta umidade de ar e elevada TGN (tabela V). De acordo Baeta (1985) e Barbosa e Silva (1995), o valor de ITGU e ITU ideal para ruminantes criados em zonas tropicais deveria ser de 74 e menor que 70 , respectivamente. O efeito de desconforto observado durante periodo experimental manisfestou-se pelo tipo de respiração que os animais apresentavam

Tabela $\boldsymbol{V}$. Monitoramento do ambiente experimental. (Monitorization of experimental environment).

\begin{tabular}{lrr}
\hline & Manhã & \\
\hline Temperate \\
Umidade relativa do ar \% & 25,3 & 28,9 \\
Temperatura do ponto de orvalho ${ }^{\circ} \mathrm{C}$ & 82,3 & 63,0 \\
Índice temperatura globo e umidade & 74,4 & 19,6 \\
Índice temperatura e umidade & 73,5 & 75,7 \\
Temperatura ambiente ${ }^{\circ} \mathrm{C}$ & 24,6 & 27,4 \\
\hline
\end{tabular}


Tabela VI. Variáveis fisiológicas em cordeiros submetidos a dietas com níveis de torta de dendê. (Physiological variables in lambs fed diets with increasing levels of palm kernel meal).

\begin{tabular}{|c|c|c|c|c|c|c|c|}
\hline & \multicolumn{4}{|c|}{ Níveis de torta de dendê (\%MS) } & \multirow{2}{*}{$\begin{array}{l}\text { CV } \\
(\%)\end{array}$} & \multirow{2}{*}{ Equação de regressão } & \multirow[t]{2}{*}{$\mathrm{R}^{2}$} \\
\hline & 0,0 & 6,5 & 13,0 & 19,5 & & & \\
\hline \multicolumn{8}{|c|}{ Freqüência respiratória } \\
\hline Manhã & 35,31 & 34,41 & 35,28 & 35,17 & 6,80 & $Y=35,04$ & - \\
\hline Tarde & 59,77 & 53,53 & 56,97 & 50,25 & 17,97 & $Y=55,13$ & - \\
\hline \multicolumn{8}{|c|}{ Temperatura retal } \\
\hline Manhã & 38,80 & 38,66 & 38,69 & 38,62 & 0,53 & $Y=38,69$ & - \\
\hline Tarde & 39,33 & 38,97 & 39,32 & 39,30 & 0,85 & $Y=39,23$ & - \\
\hline \multicolumn{8}{|c|}{ Temperatura superficial } \\
\hline Manhã & 29,38 & 29,20 & 29,66 & 29,00 & 4,43 & $Y=29,31$ & - \\
\hline Tarde & 29,04 & 28,94 & 28,98 & 28,17 & 3,41 & $Y=28,78$ & - \\
\hline \multicolumn{8}{|c|}{ Freqüência cardíaca } \\
\hline Manhã & 107,50 & 104,00 & 103,72 & 100,06 & 7,15 & $Y=107,20-0,3476 X$ & 0,921 \\
\hline Tarde & 111,53 & 107,56 & 106,95 & 101,31 & 7,60 & $Y=111,528-0,481 X$ & 0,921 \\
\hline
\end{tabular}

durante o periodo experimental que nos levou a infererir que houvesse desconforto termico uma vez que eles estavam expostos a alta humidade e temperatura ambiental.

As freqüências respiratórias, as temperaturas retais e superficiais nos dois períodos foram semelhantes para os níveis de inclusão da torta de dendê (tabela VI), provavelmente pelo fato de o estresse não ter sido muito elevado, uma vez que os animais não sofreram influência da radiação solar direta. Os valores médios de freqüência respiratória observados neste experimento foram de 35,04 e 55,13 movimentos respiratórios por minuto, valores superiores aos de referência, que são de 16 a 34 movimentos respiratórios/minuto (Swesson e Reece, 1996), o que pode ser explicado pelo alto ITGU, como mencionado anteriormente.

Entretanto, a adição da torta de dendê na dieta proporcionou comportamento linear decrescente $(\mathrm{p}<0,05)$ para freqüência cardíaca nos períodos da manhã e tarde (tabela VI). De acordo com a equação de regressão, estimou-se diminuição de 0,3476 batimentos cardíacos por minuto no período da manhã e de 0,481 batimentos no período da tarde a cada $1 \%$ de aumento do nível de torta de dendê na dieta. A substituição do farelo de milho e farelo de soja pela torta de dendê acarretou redução dos teores de carboidratos não-fibrosos (tabela I). Assim, menos carboidratos não-fibrosos no rúmen provavelmente foram fermentados e a produção do calor no organismo animal pode ter diminuído, conseqüentemente, a freqüência cardíaca dos animais também decresceu com adição dos níveis de torta de dendê na dieta.

Embora a frequência respiratória não tenha sofrido efeito da adição da torta de dendê os valores observados foram superiores aos valores de referência (16 a 34 movimentos respiratórios/minuto).

\section{CONCLUSÕES}

A inclusão da torta de dendê em até $19,5 \%$ na MS afeta o tempo de ingestão, ruminação, consumo de matéria seca, de fibra em detergente neutro e as eficiencias de ingestão e ruminação de MS e FDN.

As variáveis ambientais (temperatura do globo negro e umidade relativa do ar) observadas proporcionaram situação de desconforto térmico aos animais. 


\section{MACOME, OLIVEIRA, ARAUJO, BARBOSA, CARVALHO, GARCEZNETO E SILVA}

\section{BIBLIOGRAFIA}

AOAC. 1990. Association of analytical chemists. Official methods of analysis. $12^{\mathrm{a}} \mathrm{ed}$. Washington. 1094 pp.

Baeta, F.C. 1985. Responses of lactating dairy cows to the combined effects of temperature, humidity and wind velocity in the warm season. Thesis (PhD). University of Missouri. $218 \mathrm{pp}$.

Baeta, F.C. e Souza, C.F. 1997. Ambiência em edificações rurais: conforto animal. Universidade Federal de Viçosa. Viçosa, MG. 246 pp.

Barbosa, O.R. e Silva, R.G. 1995. Índice de conforto térmico para ovinos. Em: Reunião Anual da Sociedade Brasileira de Zootecnia, 32. Anais.. Sociedade Brasileira de Zootecnia. Brasília. pp. 141-143.

Brasil. 1998. Ministério da Agricultura e Abastecimento. Compêndio Brasileiro de Alimentação Animal. ANFAR/CBNA/SDR. São Paulo. 12 pp.

Bürger, P.J., Pereira, J.C., Queiroz, A.C., Silva, J.F.C., Valadares Filho, S.C., Cecon, P.R. e Casali, A.D.P. 2000. Comportamento ingestivo em bezerros holandeses alimentados com dietas contendo diferentes níveis de concentrado. Rev Bras Zootecn, 29: 236-242.

Buffington, D.E., Collazzo-Arocho, A. and Canton, G.H. 1981. Black globe humidity index (BGHI) as comfort equation for dairy cows. T ASAE, 24: 711-714.

Carvalho, G.G.P., Pires, A.J.V., Silva, F.F., Veloso, C.M., Silva, R.R., Silva, H.G.O., Bonomo, P. e Mendonça, S.S. 2004. Comportamento ingestivo de cabras leiteiras alimentadas com farelo de cacau ou torta de dendê. Pesqui Agropecu Bras, 39: 919-925.

Carvalho, G.G., Pires, A.J.V., Silva, F.F., Veloso, C.M., Silva, R.R. e Silva, R.R. 2006. Desempenho e digestibilidade de ovinos alimentados com farelo de cacau (Theobroma cacao L.) em diferentes niveis de substituição. Ciênc Anim Bras, 7: 115-122.

CEI. 1994. Centro de estatistica e informações. Informações básicas dos municípios baianos:
Salvador. Salvador. 440 pp.

Johnson, T.R. and Combs, D.K. 1991. Effects of prepartum diet, inert rumen bulk, and dietary polyethylene glycol on dry matter intake of lactating dairy cows. J Dairy Sci, 74: 933-944.

NRC. 1985. National Research Council. Nutrient requirements of sheep. $6^{\mathrm{a}}$ ed. National Academy of Science. Washington. 99 pp.

Penning, P.D., Rook, A.J. and Orr, R.J. 1991. Patterns of ingestive behavior of sheep continuously stocked on monocultures of ryegrass or white clover. Appl Anim Behav Sci, 31: 237-250.

Rodrigues Filho, J.A., Camarão, A.P., Batista, H.A.M., Azevedo, G.P.C. e Braga, E. 1996. Níveis de torta de dendê em substituição ao farelo de trigo no consumo voluntário e digestibilidade de concentrados. Em: Reunião da Sociedade Brasileira de Zootecnia, 35. Anais... Sociedade Brasileira de Zootecnia. Fortaleza. pp. 292-293.

Silva, H.G.O., Pires, A.J.V., Carvalho, G.G.P., Veloso, C.M. e Silva, F.F. 2008. Consumo de dietas contendo silagem de capim elefante amonizado e farelo de cacau ou torta de dende em ovinos. Rev Bras Zootecn, 37: 734-742.

Swenson, M.J. e Reece, W.O. 1996. Dukes fisiologia dos animais domésticos. $11^{\text {a }}$ ed. Guanabara Koogan. Rio de Janeiro. 855 pp.

Sniffen, C.J., Connor, J.D. and Van Soest, P.J. 1992. A net carbohydrate and protein system for evalution cattle diets. II Carbohydrate and protein availability. J Anim Sci, 70: 3562-3577.

SPSS. 2004. Statistical package for the social sciences. Release 13.0. Chicago.

Van Soest, P.J., Robertson, J.B. and Lewis, B.A. 1991. Methods for dietary fiber, neutral detergent fiber, and nonstarch polysaccharides in relation to animal nutrition. J Dairy Sci, 74: 3583-3597.

Van Soest, P.J. 1994. Nutritional ecology of the ruminant. Cornell University Press. Ithaca. 476 pp.

Archivos de zootecnia vol. 61, núm. 235, p. 342. 